\title{
Age- and gender-specific trends in respiratory outpatient visits and diagnoses at a tertiary pediatric hospital in China: a 10- year retrospective study
}

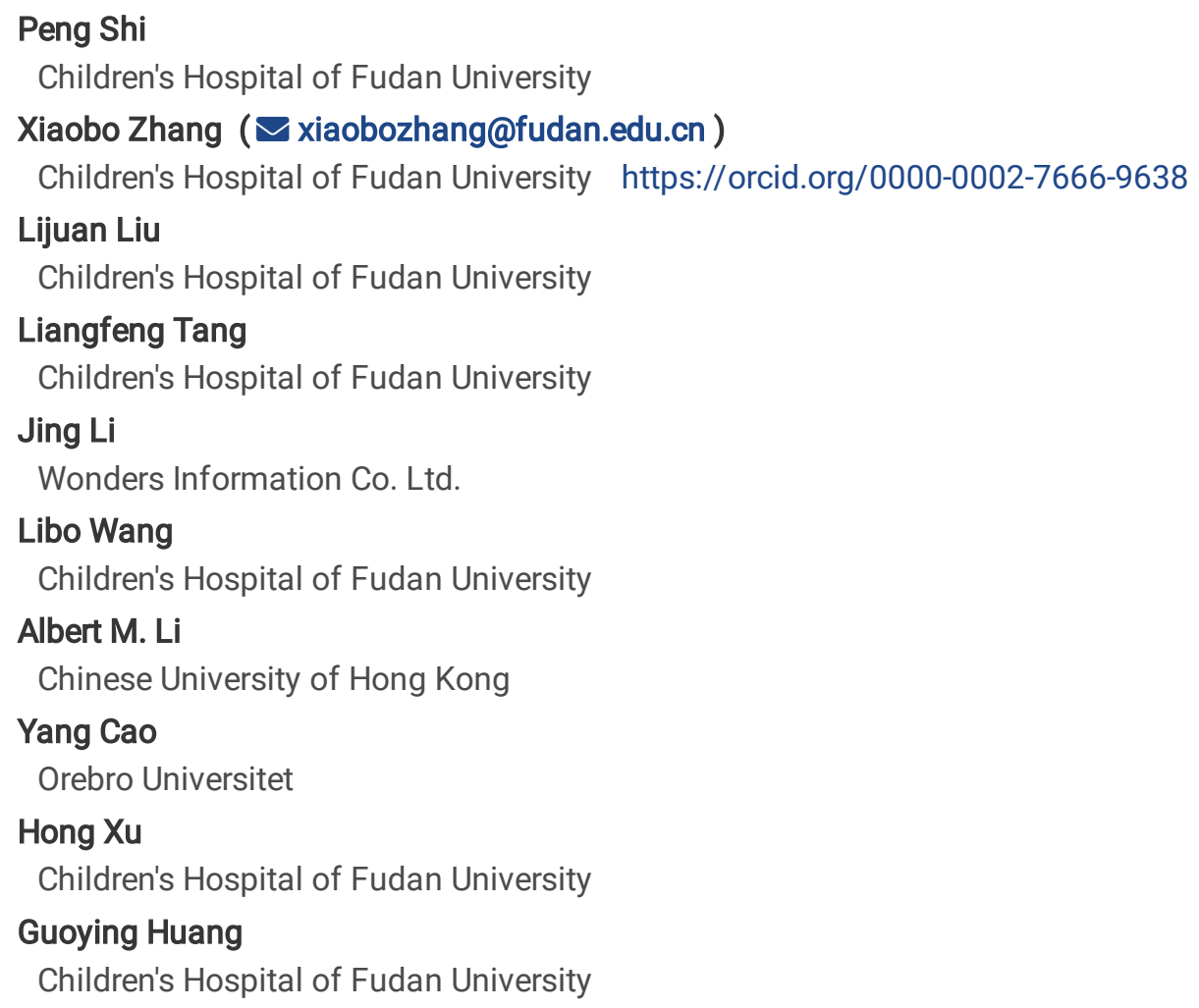

Research article

Keywords: Respiratory infections, Asthma, Pediatrics, Outpatients, Trend study

Posted Date: December 11th, 2019

DOI: https://doi.org/10.21203/rs.2.18535/v1

License: (1) This work is licensed under a Creative Commons Attribution 4.0 International License. Read Full License

Version of Record: A version of this preprint was published at BMC Pediatrics on March 12th, 2020. See the published version at https://doi.org/10.1186/s12887-020-2001-x. 


\section{Abstract}

Background: Respiratory infections are one of three leading causes of childhood mortality, and worldwide increase and recent plateau in childhood asthma has been reported. However, data on trends of respiratory diseases over long period of time is limited. This study aimed to determine the trends of respiratory disease outpatient visits (ROVs) and diagnoses (RODs) in one of the largest children's teaching hospitals in China between 2009 and 2018.

Methods: A retrospective study based on routine administrative data was designed and implemented according to the RECORD statement. Demographic details and diagnoses of the outpatients < 18 years visiting the respiratory department of the hospital were extracted from the Hospital Information System. Age- and gender-specific trends were illustrated by calculating average annual growth rate (AGR) for ROVs and comparing change of proportion for different RODs over time.

Results: There were 698054 ROVs from 285574 children (40.4\% female). Average AGR of ROVs was $15.2 \%$. Children aged 4 to $<7$ years had a faster increase than other age groups. Bronchitis (27.6\%), pneumonia (18.5\%), pneumonia affecting other systems (18.4\%), asthma and status asthmaticus (10.7\%), and vasomotor and allergic rhinitis $(9.2 \%)$ accounted for $84.4 \%$ of all RODs. The proportion of bronchitis decreased across years, with the concomitant increasing trend in the proportion of pneumonia. Age-specific trend in diagnoses showed the significantly increased proportion of asthma with ages, especially for the children aged 4 to $<18$ years. Gender-specific trend in diagnoses showed the proportion of asthma was greater for males but the average AGR was greater for females.

Conclusion: The persistent upward trend in ROVs was observed among children at different ages and a gender difference was also seen. In contrast to what has been reported, burden of asthma and allergies diseases continues to increase locally.

Keywords: Respiratory infections, Asthma, Pediatrics, Outpatients, Trend study

\section{Background}

Respiratory infections are still one of three leading causes of child mortality worldwide [1-3]. Each year childhood respiratory infections bring along a significant burden to our healthcare system in terms of manpower and resource utilization [4-6]. The National Surveillance for Asthma in the United State (US) from 1980 to 2013 reported the increasing incidence of respiratory infections and prevalence of asthma [7-9]. Compared with the report from the US, studies in Shanghai also showed an almost fivefold increased prevalence of asthma among children in China, from $2.1 \%$ in 1990 to $10.2 \%$ in 2011, with a higher prevalence of asthma in boys than in girls in the age group of 3-7 years [10]. These studies suggested age and gender differential phenomena on the increasing asthma prevalence. However, the full picture of respiratory outpatient visits (ROVs) and diagnoses (RODs) over a longer term period, especially the age- and gender-specific trends, has not been well documented, partly due to the lack of computerized databases containing diagnosis-specific outpatient data. In this study, we used 10-year routine administrative data and reported age- and gender-specific trends in ROVs and RODs among children visiting one of the largest tertiary-level pediatric hospitals in China.

\section{Methods And Materials}

\section{Study design}

This is a hospital-based retrospective study using routine administrative data and was implemented according to the RECORD statement [11]. The primary objective was to describe age- and gender-specific trends in ROVs and RODs over a 10-year period. Ethics approval was obtained from the Children's Hospital of Fudan University Ethics Committee (No. 2019 - 188).

\section{Participants}

We included visits for patients aged $<18$ years with any outpatient visit to the respiratory department of the hospital. The registration number for the visit (Visit ID) was the identification code for every visit of patient, and the patient number (Patient ID) was another identification code for different patients. We selected study population using the criteria: (1) the registration date 
between Jan 1st, 2009 and Dec 31st, 2018, (2) the registration department was respiratory department, and (3) patients aged < 18 years. We validated the data of participants in each year using the annual reports of the hospital.

\section{Setting}

The setting of this study is the Children's Hospital of Fudan University, a tertiary, teaching hospital and a National Children's Medical Center located in Shanghai, China.

\section{Data sources and data linkage}

Our data are based on linking hospital outpatient registration data in the health information system (HIS) from 2009-2018 to diagnosis records in different databases during the three upgrading phases of the HIS: backup of diagnosis data for outpatient department(2009-2012), back transcription of diagnosis data in registry database (2012-2017), and diagnosis data in Outpatient Department Entry System(2017-2018) for all patients who visited the respiratory department in the hospital. The participant selection and data linkage process is shown in Fig. 1.

\section{Variable coding and grouping}

(1) Outpatient visit The Visit ID is unique in the HIS for every visit of patient.

(2) Age categories of patient Age of patient was calculated by the difference between birth date and visit date and classified as $<1$ year, 1 to $<4$ years, 4 to $<7$ years, 7 to $<12$ years, and 12 to $<18$ years.

(3) Visit diagnoses Each visit recorded in the HIS system includes outpatients diagnoses, with the first being considered the primary diagnosis. Diagnoses are coded by the International Classification of Diseases, 10th Revision (ICD-10). We selected the primary diagnosis for each visit and categorized the diagnoses based on the category codes (Appendix 1).

\section{Data quality}

Completeness and distribution were assessed and compared for key variables (age, gender, and diagnosis). Missing rates of key variables were listed in Appendix 2 and distribution comparison was used to assess the bias due to missing data.

\section{Statistical methods}

We used SQL server 2010 and Python platform for data processing and IBM SPSS Statistics 22.0 for data analysis. For descriptive analyses, number and percentage (\%) was used for qualitative variables, median and interquartile range (IQR) or mean \pm SD for continuous variables according to its distribution. We analyzed the 10-year trends using two indicators, including the number of ROVs and the proportion of RODs. The trend in ROVs was assessed by average annual growth rate (AGR) to show the constant ratio through the studied period. The average AGR was calculated in terms of a geometric growth expressed as $\left(X_{n} / X_{0}\right)^{(1 / n)}-1$ (where $X_{0}$ is the value of the baseline and $X_{n}$ is the value of the $n$th year)) [12]. A simple linear regression was used to model the increasing trend in ROVs with year. The change in RODs was compared by the proportion of the selected diagnosis to the whole RODs. Chi-squared test for trend was used to test whether there is a systematic increase or decrease in the proportions through the years. Distribution of patients with missing data through the years was compared using the chi-squared test. A two-tailed $P$ value of less than 0.05 was considered statistically significant. Sample sizes were large in this study, therefore effect sizes were more meaningful than $P$ value for trend analysis and distribution comparisons [13].

\section{Results}

\section{Patient characteristics}


Total of 285574 patients attended to the respiratory outpatient department of the hospital during the 10-year study period.

Preschool children aged $<7$ years old accounted for $88.0 \%$ of all the children. The number of children aged 1 to $<4$ years was twice of those aged $<1$ years and 4 to $<7$ years. Males accounted for $59.6 \%$ of the patient population, $39.4 \%$ of the patients were covered by government insurance and $60.6 \%$ were self-finance. Respiratory specialist clinic provided medical service for $46.5 \%$ patients mainly with special respiratory diseases. The doctors with associate professor position or above provided medical service for $52.3 \%$ patients in the expert-clinic. The median and the 25 th and the 75 th percentiles of visits per children were 1 and $(1,3)$, and the number of visits had no significant difference between the patients covered by government insurance and those who were selffinance $[1(1,3)$ vs. $1(1,2)]$. (Table 1) 
Table 1

Characteristics of patients, 2009-2018

\section{Patients visited the respiratory outpatient department}

$\mathrm{n}$ of patients

Age, $n(\%)$

$<1$ year

1 to $<4$ years

4 to $<7$ years

7 to $<12$ years

12 to $<18$ years

Total ${ }^{[1]}$

Sex, $\mathrm{n}(\%)$

Male

Female

Payer type, $n(\%)$

Government insurance

Self-finance

Clinic types, $\mathrm{n}(\%)$

Specialist Clinic

Expert-clinic

Disease-specific Clinic

Total ${ }^{[2]}$

Average visits per patients

Government insurance

Mean \pm SD

$\operatorname{Median}\left(\mathrm{P}_{25}, \mathrm{P}_{75}\right)$

Self-pay

Mean \pm SD

$\operatorname{Median}\left(\mathrm{P}_{25}, \mathrm{P}_{75}\right)$

Total

Mean \pm SD

$\operatorname{Median}\left(\mathrm{P}_{25}, \mathrm{P}_{75}\right)$
74034(23.0)

132178(41.1)

76818(23.9)

33825(10.5)

4512(1.4)

321368

170192(59.6)

115382(40.4)

112495(39.4)

173079(60.6)

170808(46.5)

192081(52.3)

4434(1.2)

367323

$1(1,3)$

$2.1 \pm 2.7$

$1(1,2)$

$2.4 \pm 3.3$

$1(1,3)$

Note: [1] A child could contribute data to multiple age groups as he or she visited the department of respiratory disease in different year.

[2] A child could contribute data to multiple clinic types as he or she visited the department of respiratory disease in different type. 


\section{Overall trend in ROVs}

During the 10-year study period, the overall ROVs were 698054 with an average AGR of $15.2 \%$. The AGR stayed stable around $10 \%$ after 2013. (Fig. 2)

\section{Age trends in ROVs}

Figure 3 displays the change of the proportions and trends in number of visits at different age groups through the years. For children aged $<1$ year, the proportion of total visits declined from $24.3 \%$ in 2013 to $17.4 \%$ in $2018(P<0.001)$ and the annual visits increased over time with an average AGR of $14.7 \%(P<0.001)$. For children aged 1 to $<4$ years, they remained stable $43 \%$ of total visits and increased with an average AGR of $14.9 \%$. For children aged 4 to $<7$ years, the proportion of total visits increased from 22.8-28.4\% $(P<0.001)$ and the average AGR appeared the highest of $16.9 \%$ among all age groups $(P<0.001)$. For children aged 7 to $<12$ years and 12 to $<18$ years, they accounted for $9 \%$ and $1 \%$ of total visits and the average AGR increased slowly from $13.1-$ $13.8 \%$, respectively.

\section{Gender trends in ROVs}

For male children, they accounted for $60.6 \%$ of total visits, from $62.9 \%$ in 2009 to $59.2 \%$ in 2018 , with an average AGR of $14.4 \%$. For female children, the proportion to total visits varied from $37.1 \%$ in 2009 to $40.8 \%$ in 2018 with an average AGR of $16.4 \%$.

\section{Overall diagnoses distribution}

During the past 10 years, 23 different diagnoses categories were identified for all ROVs. Bronchitis was the most commonly identified diagnosis with 190088 (27.6\%) of total visits, followed by pneumonia (126 887, 18.5\%), pneumonia affecting other systems (126 469, 18.4\%), asthma and status asthmaticus (73 443, 10.7\%), vasomotor and allergic rhinitis (62 951, 9.2\%), other lower respiratory infections (42 353,6.2\%), and other respiratory diseases $(65261,9.4 \%)$. The distributions of all the identified diagnoses are shown in Fig. 4.

\section{Gender- and age-specific trends in proportion of diagnoses}

Figure 5 shows the change of proportions of the selected three main diagnoses, bronchitis, pneumonia, asthma and status asthmaticus, stratified by gender.

For male children, the proportion of bronchitis in all ages declined from $34.8 \%$ in 2009 to $25.6 \%$ in 2018. In different age categories, the proportion of bronchitis for children aged $<1$ year declined from $34.5-24.8 \%$, aged 1 to $<4$ years $37.1-27.9 \%$, aged 4 to $<$ 7 years $34.5-25.7 \%$, aged 7 to $<12$ years $33.4-19.5 \%$, and aged 12 to $<18$ years $26.1-11.3 \%$. For female children, the proportion of bronchitis in all ages and age-specific categories showed the similar decline through the years.

For male children diagnosed pneumonia, the proportion of the disease in all ages increased from $13.0 \%$ in 2009 to $21.7 \%$ in 2018 . Comparing the different age categories, children aged $<1$ year and 1 to $<4$ years accounted for larger proportion of pneumonia (13.6\% and $11.96 \%$ in 2009; and $22.1 \%$ and $22.4 \%$ in 2018) than the older age categories, for example, male children aged 4 to $<7$ years, $7<12$ years, and 12 to $<18$ years $(4.8 \%, 8.8 \%$, and $7.4 \%$ in 2009 ; and $12.9 \%, 13.2 \%$, and $12.7 \%$ in 2018 ). For female children, the proportion of pneumonia showed the similar increasing trend through the years.

For male children diagnosed asthma and status asthmaticus, the age differential phenomena were evident. Through the past 10 years, the proportion of asthma increased by age categories. Male children aged $<1$ year and 1 to $<4$ years had lower proportion ( $15.9 \%$ and $12.9 \%$ in 2009 ; and $12.1 \%$ and $8.2 \%$ in 2018 , respectively) compared to the other age categories: aged 4 to $<7$ years (20.4\% in $2009 ; 17.3 \%$ in 2018$)$, age 7 to < 12 years (24.9\% in $2009 ; 22.7 \%$ in 2018$)$, and aged 12 to < 18 years $(35.1 \%$ in $2009 ; 28.6 \%$ in 2018). For female children, the proportion of asthma was lower than the male children at every age categories with similar change through the years. For example, $17.4 \%$ female children aged 7 to $<12$ years were diagnosed asthma in 2009 and $13.6 \%$ in 


\section{Gender- and age-specific trends in visits of diagnoses}

Table 2 showed the trends of the number of visits by respiratory diagnoses. For children diagnosed with bronchitis, the females had higher average AGR compared to the males in all age categories ( $14.6 \%$ vs. 10.39 for aged $<1 \mathrm{y}, 12.9 \%$ vs. $10.8 \%$ for aged 1 to $<4$ years, $15.5 \%$ vs. $12.4 \%$ for aged 4 to $<7$ years, $7.4 \%$ vs. $6.0 \%$ for aged 7 to $<12$ years, and $6.8 \%$ vs. $2.5 \%$ for aged 12 to $<18$ year). Children aged $<7$ years have higher average AGR than the older children for both the males and females $(P$ for trend $<0.001)$.

Table 2

Age- and gender-specific trends in average AGR of visits by diagnosis, 2009-2018

\begin{tabular}{|c|c|c|c|c|c|c|c|c|c|c|}
\hline & \multicolumn{2}{|l|}{$<1 \mathrm{y}$} & \multicolumn{2}{|l|}{$1 \mathrm{y} \sim$} & \multicolumn{2}{|l|}{$4 y \sim$} & \multicolumn{2}{|l|}{$7 y \sim$} & \multicolumn{2}{|c|}{$12 \sim<18 y$} \\
\hline & $\begin{array}{l}\text { Average } \\
\text { AGR } \\
(\%)\end{array}$ & $\begin{array}{l}\mathrm{P} \text { for } \\
\text { trend }\end{array}$ & $\begin{array}{l}\text { Average } \\
\text { AGR } \\
(\%)\end{array}$ & $\begin{array}{l}\mathrm{P} \text { for } \\
\text { trend }\end{array}$ & $\begin{array}{l}\text { Average } \\
\text { AGR } \\
(\%)\end{array}$ & $\begin{array}{l}\mathrm{P} \text { for } \\
\text { trend }\end{array}$ & $\begin{array}{l}\text { Average } \\
\text { AGR } \\
(\%)\end{array}$ & $\begin{array}{l}\mathrm{P} \text { for } \\
\text { trend }\end{array}$ & $\begin{array}{l}\text { Average } \\
\text { AGR } \\
(\%)\end{array}$ & $\begin{array}{l}\mathrm{P} \text { for } \\
\text { trend }\end{array}$ \\
\hline \multicolumn{11}{|c|}{ Bronchitis } \\
\hline Male & 10.39 & 0.004 & 10.77 & 0.003 & 12.38 & $\begin{array}{l}< \\
0.001\end{array}$ & 6.01 & 0.008 & 2.51 & 0.105 \\
\hline Female & 14.62 & $\begin{array}{l}< \\
0.001\end{array}$ & 12.90 & 0.001 & 15.49 & $\begin{array}{l}<.001 \\
0.01\end{array}$ & 7.37 & 0.002 & 6.75 & 0.066 \\
\hline \multicolumn{11}{|c|}{ Pneumonia } \\
\hline Male & 20.84 & $\begin{array}{l}< \\
0.001\end{array}$ & 22.62 & $\begin{array}{l}<.001 \\
0.00\end{array}$ & 21.25 & $\begin{array}{l}<.001 \\
0.001\end{array}$ & 17.61 & $\begin{array}{l}<.001 \\
0.001\end{array}$ & 19.58 & $\begin{array}{l}< \\
0.001\end{array}$ \\
\hline Female & 23.98 & $\begin{array}{l}< \\
0.001\end{array}$ & 22.30 & $\begin{array}{l}< \\
0.001\end{array}$ & 23.17 & $\begin{array}{l}< \\
0.001\end{array}$ & 21.06 & $\begin{array}{l}< \\
0.001\end{array}$ & 27.03 & $\begin{array}{l}<.001 \\
0.00\end{array}$ \\
\hline \multicolumn{11}{|c|}{ Asthma and status asthmaticus } \\
\hline Male & 11.10 & 0.001 & 8.79 & 0.038 & 13.99 & 0.002 & 11.37 & 0.001 & 9.95 & 0.001 \\
\hline Female & 12.12 & $\hat{0}_{0.001}$ & 10.11 & 0.009 & 18.06 & 0.001 & 11.24 & $<.001$ & 18.43 & 0.001 \\
\hline
\end{tabular}

For children diagnosed with pneumonia, they had nearly two fold average AGR compared to the children diagnosed with bronchitis and asthma. Female children had also higher average AGR than the males in all age categories, and the average AGR varied little between different age categories.

For children diagnosed with asthma and status asthmaticus, the average AGR was similar to that of the children with bronchitis, while it was higher than that of children with bronchitis aged $>=7$ years. For each age category, female children with asthma had higher average AGR compared to the male children $\left(P_{s}<0.001\right)$.

\section{Discussion}

Our study provided real world data of secular trends in outpatient visits and diagnosis amongst children visiting the one of the largest children's hospitals in China. Detailed computer records of this large patient population allowed investigating age- and gender-specific trends over a period of 10 years.

During the past 10 years, the trend in ROVs increased rapidly in the early years of the decade and slowed down substantially after 2013. The result suggests a great increase in the demand of specialist medical service for respiratory diseases in the early years and the supply of respiratory outpatient service nearly reaching the full capacity in the subsequent years. 
Among the total ROVs, preschool children (aged $<7$ years) were the main consumers receiving specialist medical services. Children aged 1 to $<4$ years accounted for $43.0 \%$ of ROVs in total, 4 to $<7$ years accounted for a further $24.2 \%$, and $<1$ year accounted for $22.6 \%$, respectively. The higher averaged AGR of the preschool children compared to the school children (aged 7 to $<18$ years) also shown the similar trends in number of visits. This is understandable as preschoolers, with relatively naïve immune system, are the most susceptible to respiratory diseases and different environmental triggers. For gender trends in ROVs, boys were more susceptible to respiratory diseases compared to girls under the assumptions on stable population and sex ratio in the catchment areas of the hospital. This can be explained by that the sex-based DNA methylation signatures at birth and human respiratory conditions are largely influenced by sex resulting in overall higher risk for males than for females, particularly during early life [14].

When we further analyzed the trends in diagnoses of respiratory diseases, we found that bronchitis, pneumonia, asthma and status asthmaticus were main RODs, accounting for more than $80 \%$ of total visits during the past 10 years. We explored the age- and gender-specific trends in number of visits and proportions of the selected diagnoses. The proportion of bronchitis decreased through the years, with a concomitant increasing trend in proportion of pneumonia. The disparity between ages indicates that preschool children had higher proportion of bronchitis or pneumonia compared to school children, and there were no statistically significant difference between males and females. Particularly when comparing the average AGRs of number of visits between males and females, we found consistently higher average AGRs of female children than of male children in all age categories (Table 2). Although males are more likely to be affected by infection-induced acute inflammation compared to females, the increasing trends in infection-induced respiratory disease burden for females should be aware of by pediatric physicians and policy makers [15].

For outpatient children diagnosed with asthma and status asthmaticus, we found a U-shape time trend of the proportion of asthma, which decreased till 2015 and then increased till 2018. The variation of proportion of asthma remained in the range from 5-10\% for all children. In China, guideline for the diagnosis and optimal management of asthma in children were first published in 2008 and then updated in $2016[16,17]$. The publication of the criteria and awareness of asthma management influenced the variation of proportion of asthma in ROVs. The differential age- and gender-specific trends in proportion of asthma were observed in our study. The proportion of asthma increased with age significantly and consistently for both males and females. This finding was in accordance to the previous studies in the US, Europe, and China. The prevalence of asthma across all ages was $21.8 \%$ in Philadelphia, with an increased prevalence after $6-10$ years of ages (22.9\%) and to a peak between 14 and 17 years of age (23.0\%) from 2001 to 2013[18]. In Northern Sweden, the prevalence of physician-diagnosed asthma increased from 5.7\% at age of 7-8 to $7.7 \%$ at age of $11-12(P<0.001)$ [19]. In Shanghai, China, the prevalence of childhood asthma showed greatly increasing from 1990 to 2011, and asthma was more reliably diagnosed in school children [10]. Asthma was in the process of increasing and the age of 4 to $<7$ years is a critical period for pediatric physicians to take an early action on the treatment of asthma and allergic diseases. Considering the gender-specific trend in proportion and number of visits of asthma, our study shows the higher proportion of asthma in males than in females, while the greater average AGR among females. The similar findings were also observed in the latest report in the US from 2001 to 2013. The US survey suggested asthma prevalence is higher among males in 5-9 years of age and increases among females compared to males in 10-17 years of age [9]. This gender-specific trend suggests the 'adolescent switch' discussed in other studies. Asthma mostly affects boys in childhood and women in adulthood[20], with many factors contributing to its frequency and severity, including shift in sex hormone[21-23], genetic factors[24], maternal asthma[25], and environmental exposure[26]. Gender-specific trend for asthma should be taken into account in asthma management and it is relevant to consider gender differences in the daily clinical practice [27].

\section{Strengths And Limitations}

One of the strengths of our study is the substantial large sample size of the 10-year administrative data. Second, we reported strictly in accordance to the RECORD statement. Last but not least, we used the ICD-10 classification system to analyze the age and gender-specific trend in RODs, which makes comparison with other studies possible.

There are also limitations in our study. Firstly, this is a hospital-bases study in a single center. We cannot calculate the prevalence of pediatric RODs in the population. Therefore, we assumed the population and sex ratio stable in the catchment areas of the hospital (Appendix 3). Comparing the proportion of respiratory diseases can be approximate to comparing the prevalence of the diseases. Secondly, the nature of retrospective study has a risk of report bias. The diagnosis recorded in the HIS system may have a higher 
occurrence of unspecified diagnosis and use of the ICD-10 category symptoms, signs and abnormal clinical and laboratory findings in the early of the decade. Thirdly, the bias derived from missing data in diagnosis cannot be excluded. We compared the distribution of age and gender of patients with missing data in diagnosis and no systematic difference was found (Appendix 2). Lastly, our findings are based on data from a tertiary pediatric hospital, which may not be generalized to other suppliers of the pediatric medical service.

\section{Conclusion}

Our findings suggest that the upward trend in pediatric ROVs among children at different ages in the past 10 years. More attention should be given to the children aged more than 4 years and between 'adolescent switch' who present an increasing trend of asthma. Gender differences and long-term prevention to control inflammation should be taken into account in daily clinical practice.

\section{Abbreviations}

ROVs:respiratory outpatient visits; RODs:respiratory outpatient diagnoses; RD:respiratory disease; HIS:Hospital Information System; AGR:annual growth rate; ICD-10:the International Classification of Diseases, 10th Revision; IQR:interquartile range

\section{Declarations}

\section{Funding}

This study was funded by Shanghai Science and Technology Committee (grand number: 18411951700), and by Intelligent Medical Research Project of Shanghai Health and Family Planning Commission (grand number: 2018ZHYL0225)

\section{Authors' Contributors}

Prof Zhang conceptualized and designed the study, supervised all analyses, critically reviewed and revised the manuscript.

Mr Shi designed the study, coordinated and supervised data collection, conducted the data analyses, drafted and revised the manuscript.

Drs Liu, Tang assisted in data clearing, interpretation of the results and revised the manuscript.

Ms J. Li designed the data collection instruments, collected data, carried out the initial analyses.

Drs A.M. Li, Wang, $\mathrm{Xu}$, and Huang critically reviewed the manuscript for important intellectual content.

Prof Cao critically reviewed the manuscript for study design and statistical analysis.

All authors approved the final manuscript as submitted and agreed to be accountable for all aspects of the work.

\section{Consent for publication}

Not applicable

\section{Competing interest}

The authors declare that they have no competing interests.

\section{Acknowledgments}

The authors thank Yu Shi, Wenjie Zheng, Jie Tao, Yong Shi (Wonders Information System Co. Ltd.) for the support of data extraction and analysis. Chengjie Ye, Yimin Lao (Department of Information Management, Children's Hospital of Fudan University) for the help of setting up the system environment for data analysis.

\section{References}


1. Liu L, Oza S, Hogan D, et al. Global, regional, and national causes of child mortality in 2000-13, with projections to inform post2015 priorities: an updated systematic analysis. Lancet. 2015;385(9966):430-40.

2. Liu L, Johnson HL, Cousens S, et al. Global, regional, and national causes of child mortality: an updated systematic analysis for 2010 with time trends since 2000. Lancet. 2012;379(9832):2151-61.

3. He C, Liu L, Chu Y, et al. National and subnational all-cause and cause-specific child mortality in China, 1996-2015: a systematic analysis with implications for the Sustainable Development Goals. Lancet Glob Health. 2017;5(2):e186-97.

4. Chen J, Hu P, Zhou T, et al. Epidemiology and clinical characteristics of acute respiratory tract infections among hospitalized infants and young children in Chengdu, West China, 2009-2014. Bmc Pediatr. 2018;18(1):216.

5. Gooskens J, van der Ploeg V, Sukhai RN, et al. Clinical evaluation of viral acute respiratory tract infections in children presenting to the emergency department of a tertiary referral hospital in the Netherlands. Bmc Pediatr. 2014;14:297.

6. Yang J, Jit M, Leung KS, et al. The economic burden of influenza-associated outpatient visits and hospitalizations in China: a retrospective survey. Infect Dis Poverty. 2015;4:44.

7. Moorman JE, Rudd RA, Johnson CA, et al. National surveillance for asthma-United States, 1980-2004. MMWR Surveill Summ. 2007;56(8):1-54.

8. Moorman JE, Akinbami LJ, Bailey CM, et al. National surveillance of asthma: United States, 2001-2010. Vital Health Stat 3. 2012(35):1-58.

9. Akinbami LJ, Simon AE, Schoendorf KC. Trends in allergy prevalence among children aged 0-17 years by asthma status, United States, 2001-2013. J Asthma. 2016;53(4):356-62.

10. Huang C, Liu W, Hu Y, et al. Updated prevalences of asthma, allergy, and airway symptoms, and a systematic review of trends over time for childhood asthma in Shanghai, China. Plos One. 2015;10(4):e121577.

11. Benchimol El, Smeeth L, Guttmann A, et al. The REporting of studies Conducted using Observational Routinely-collected health Data (RECORD) statement. Plos Med. 2015;12(10):e1001885.

12. Data and metadata reporting and presentation handbook: OECD Organization For Economic Development; 2007.

13. Lin M, Lucas HC, Shmueli G. Research Commentary-Too Big to Fail: Large Samples and the p-Value Problem. Inform Syst Res. 2013;24(4):906-17.

14. Nino CL, Perez GF, Isaza N, et al. Characterization of Sex-Based Dna Methylation Signatures in the Airways During Early Life. Sci Rep. 2018;8(1):5526.

15. Chamekh M, Deny M, Romano M, et al. Differential Susceptibility to Infectious Respiratory Diseases between Males and Females Linked to Sex-Specific Innate Immune Inflammatory Response. Front Immunol. 2017;8:1806.

16. The Subspecialty Group of Respiratory Diseases, The Society of Pediatrics, Chinese Medical Association, Editorial board of Chinese Journal of Pediatrics. Guideline for the diagnosis and optimal management of asthma in children. Chin J Pediatr. 2008;46(10):745-53.

17. The Subspecialty Group of Respiratory Diseases, The Society of Pediatrics, Chinese Medical Association, Editorial board of Chinese Journal of Pediatrics. Guideline for the diagnosis and optimal management of asthma in children (2016). Chin J Pediatr. 2016;54(3):167-81.

18. Hill DA, Grundmeier RW, Ram G, et al. The epidemiologic characteristics of healthcare provider-diagnosed eczema, asthma, allergic rhinitis, and food allergy in children: a retrospective cohort study. Bmc Pediatr. 2016;16:133.

19. Bjerg A, Ronmark E. Asthma in school age: prevalence and risk factors by time and by age. Clin Respir J. 2008;2 Suppl 1:12326.

20. Nowak-Wegrzyn A, Ellis A, Castells M. Sex and allergic diseases. Ann Allergy Asthma Immunol. 2019;122(2):134-35.

21. Keselman A, Fang X, White PB, et al. Estrogen Signaling Contributes to Sex Differences in Macrophage Polarization during Asthma. J Immunol. 2017;199(5):1573-83.

22. Fuseini H, Newcomb DC. Mechanisms Driving Gender Differences in Asthma. Curr Allergy Asthma Rep. 2017;17(3):19.

23. Chen W, Mempel M, Schober W, et al. Gender difference, sex hormones, and immediate type hypersensitivity reactions. Allergy. 2008;63(11):1418-27. 
24. Arathimos R, Granell R, Haycock P, et al. Genetic and observational evidence supports a causal role of sex hormones on the development of asthma. Thorax. 2019.

25. Tse SM, Coull BA, Sordillo JE, et al. Gender- and age-specific risk factors for wheeze from birth through adolescence. Pediatr Pulmonol. 2015;50(10):955-62.

26. Strong C, Chang LY. Family socioeconomic status, household tobacco smoke, and asthma attack among children below 12 years of age: gender differences. J Child Health Care. 2014;18(4):388-98.

27. Ciprandi G, Gallo F. The impact of gender on asthma in the daily clinical practice. Postgrad Med. 2018;130(2):271-73.

\section{Figures}

Linked the diagnosis database -Backup of diagnosis data for outpatients department, 2009-2012 -Back transcription of diagnosis data in registry database, 2012-2017 -Diagnosis data in Outpatient Department Entry System, 2017-2018

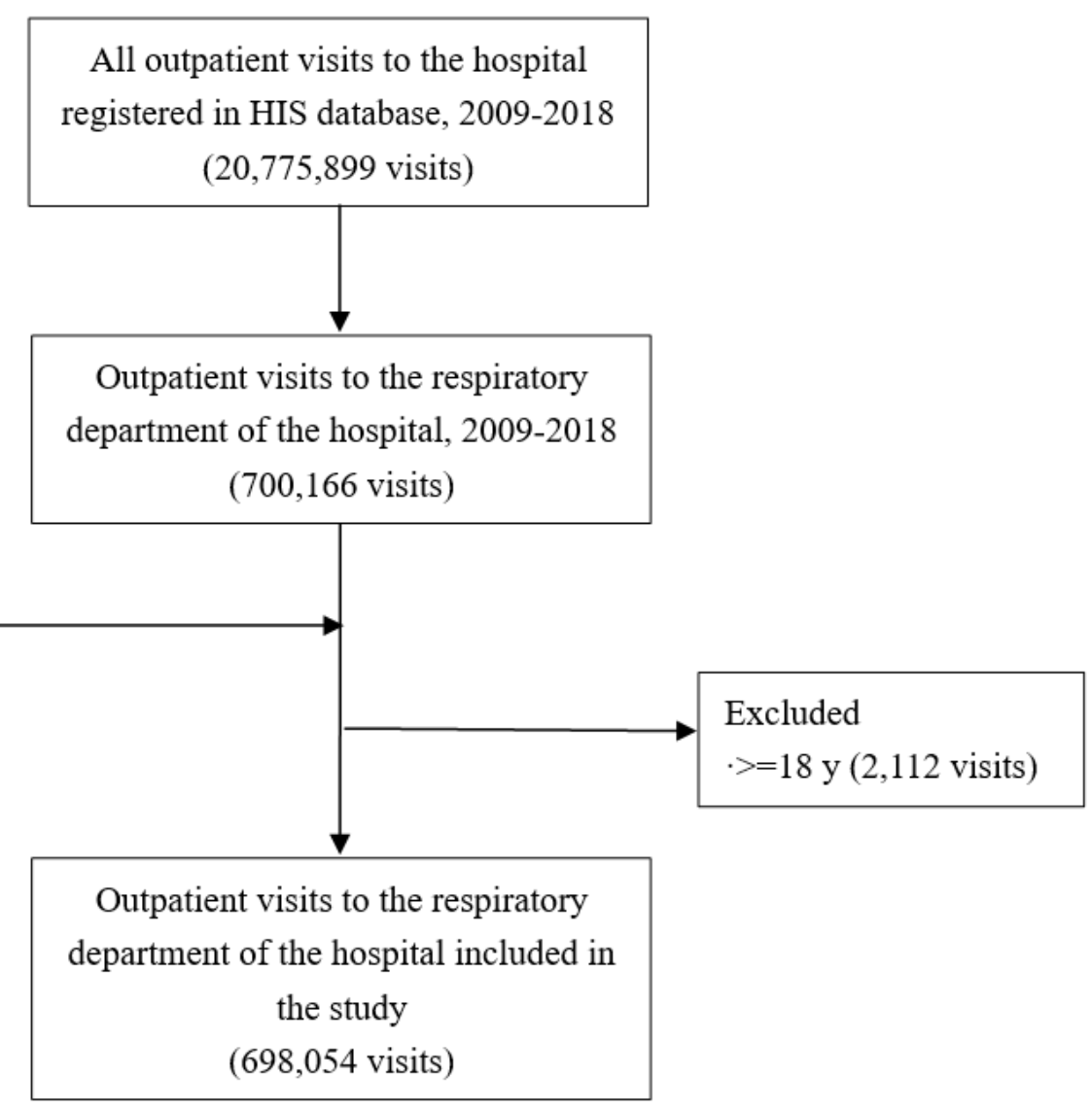

Figure 1

Flowchart illustrating selection of participants and data linkage process 


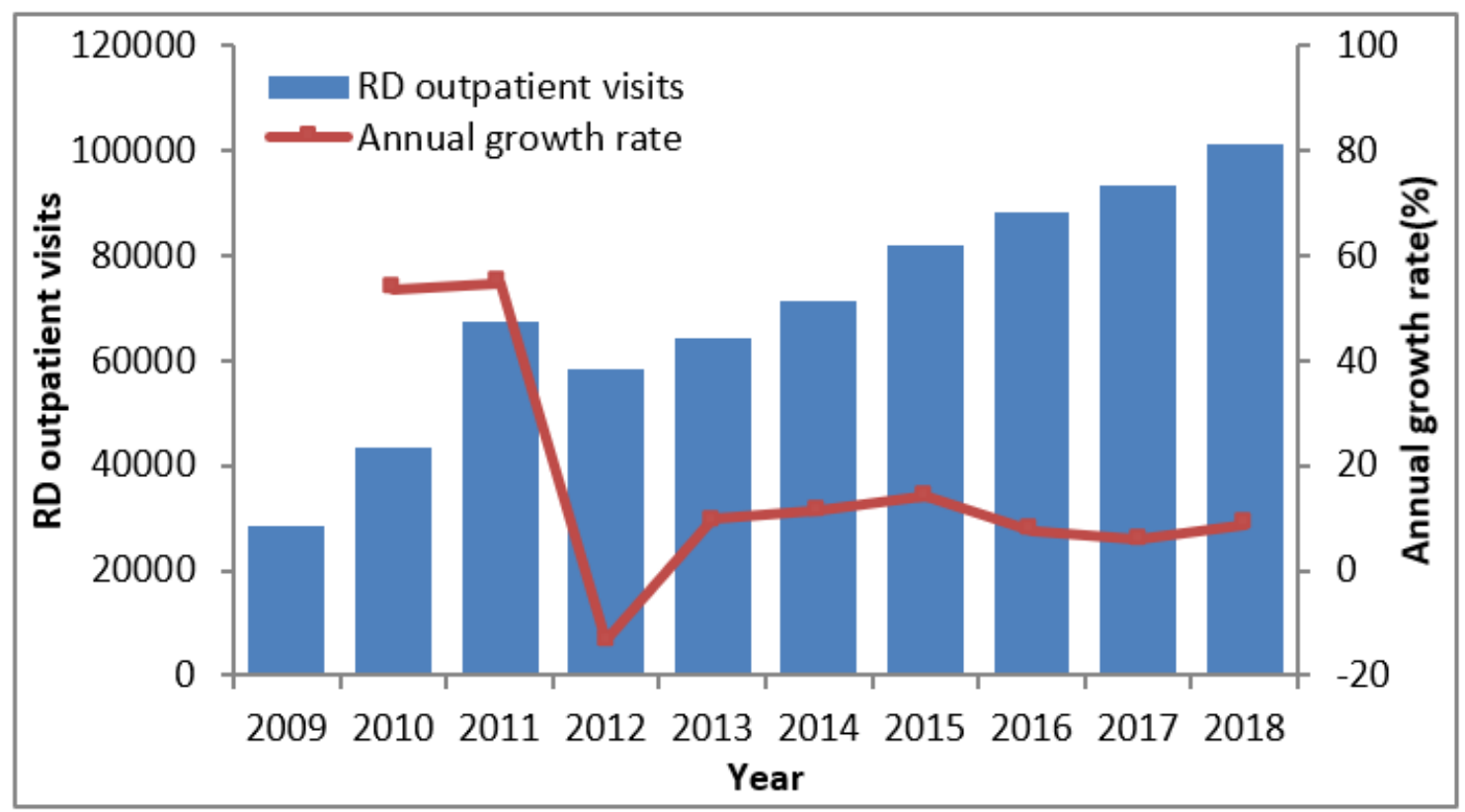

\section{Figure 2}

Overall trend in respiratory outpatient visits, 2009-2018
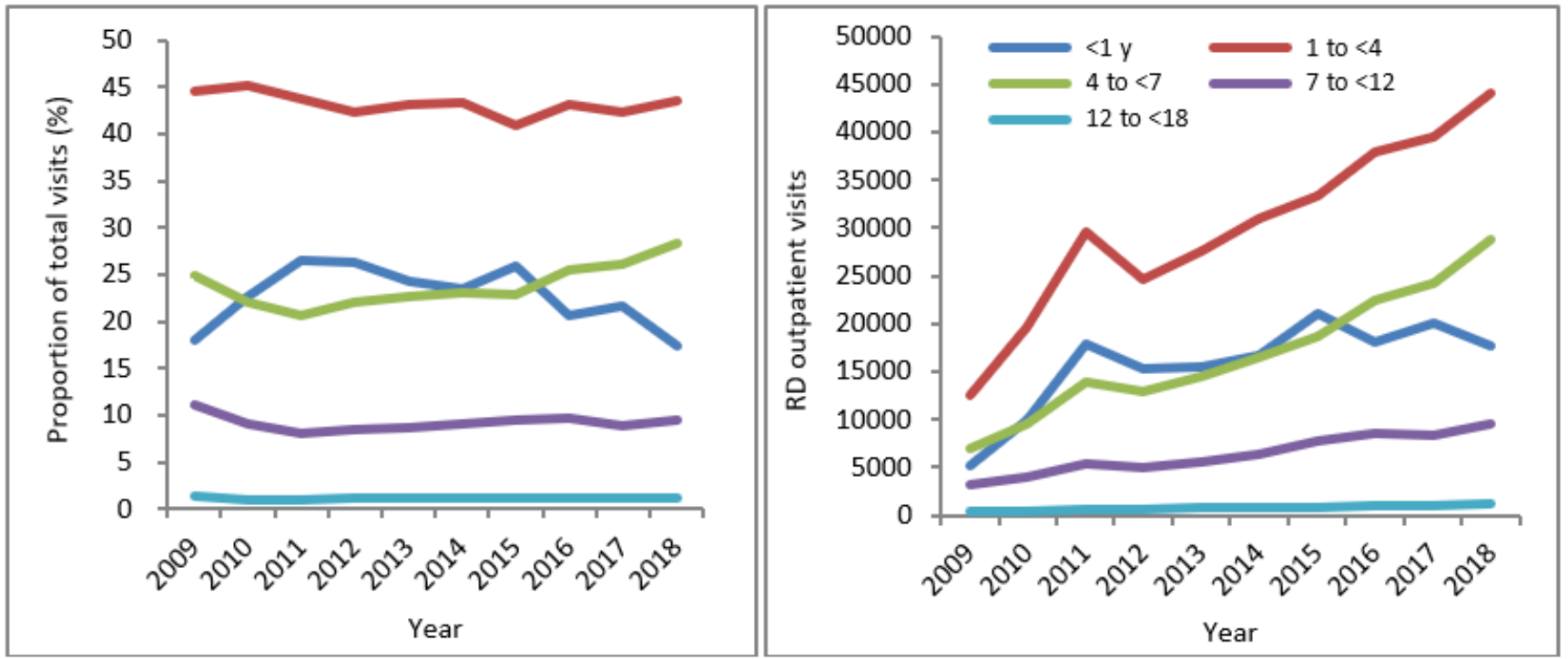

Figure 3

Age trends in proportion and visits of respiratory outpatient, 2009-2018 


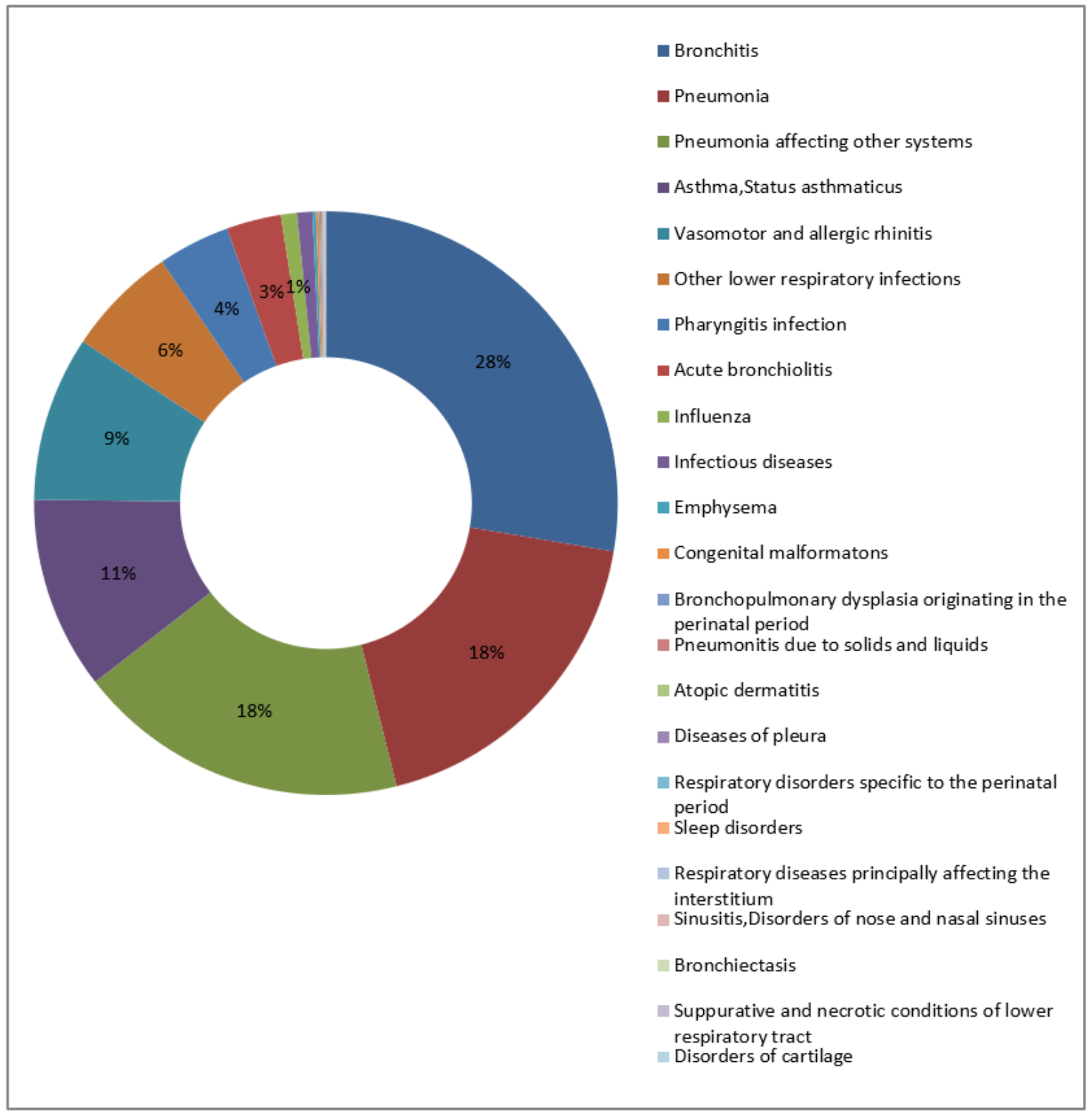

Figure 4

Distribution of diagnoses of respiratory diseases, 2009-2018 

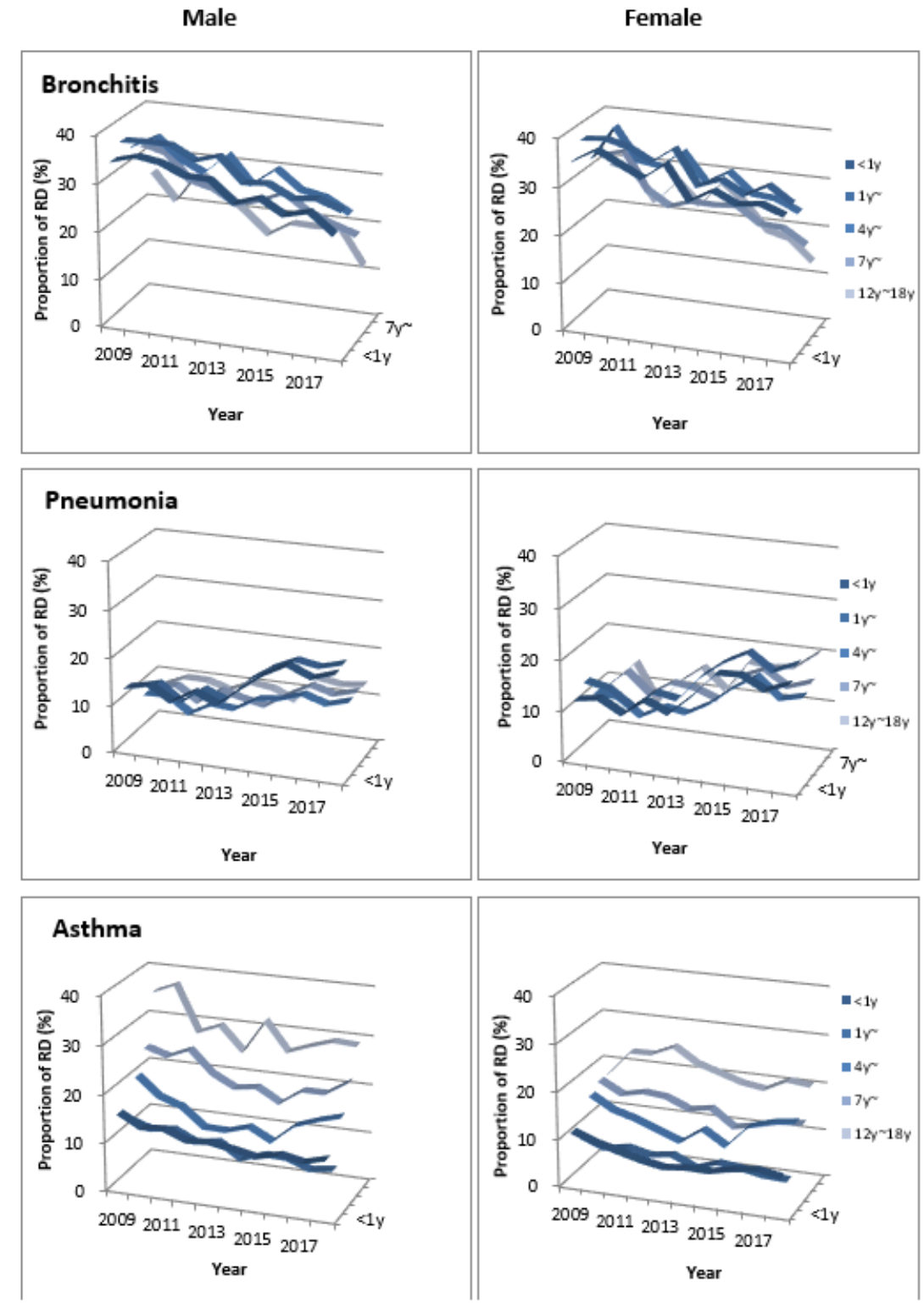

Figure 5

Age and gender trends in proportion of respiratory diagnoses, 2009-2018 